\title{
Efecto de la suplementación con semilla de algodón y maíz en el desempeño productivo y calidad de la carne de ovinos
}

\section{Effect of supplementation with cottonseed and corn on the productive performance and quality of sheep meat}

\author{
Emiro Suárez-Paternina'; Libardo Maza-Angulo²; Lorena Aguayo-Ulloa ${ }^{3}$; Oscar Vergara-Garay ${ }^{4}$; \\ Wilson Barragán-Hernández ${ }^{5}$; Moris Bustamante-Yánez ${ }^{6}$
}

\begin{abstract}
'Zoot., M.Sc. Corporación Colombiana de Investigación Agropecuaria - Agrosavia, Centro de Investigación Turipaná. Montería - Córdoba, Colombia; e-mail: esuarez@agrosavia.co; (1) http://orcid.org/0000-0003-2271-7160
\end{abstract}

${ }^{2}$ MVZ, M.Sc. Universidad de Córdoba, Facultad de Medicina Veterinaria y Zootecnia. Montería - Córdoba, Colombia; e-mail: Imaza@correo.unicordoba.edu. co; (D) http://orcid.org/0000-0002-8148-8532

${ }^{3}$ MVZ, Ph.D. Corporación Colombiana de Investigación Agropecuaria - Agrosavia, Centro de Investigación Turipaná. Montería - Córdoba, Colombia; e-mail: laguayo@agrosavia.co; (D) https://orcid.org/0000-0002-3825-9515

${ }^{4}$ Zoot, Ph.D. Universidad de Córdoba, Facultad de Medicina Veterinaria y Zootecnia, Grupo de Investigación en Producción Animal Tropical. Montería Córdoba, Colombia; e-mail: overgara@correo.unicordoba.edu.co; (D) http://orcid.org/0000-0002-1027-9469

${ }^{5}$ Zoot, MSc, Ph.D. Corporación Colombiana de Investigación Agropecuaria - Agrosavia, Centro de Investigación Turipaná. Córdoba, Colombia. e-mail: wbarraganh@agrosavia.co; (D) http://orcid.org/0000-0003-3528-4296

${ }^{6}$ MVZ, M.Sc. Universidad de Córdoba, Facultad de Medicina Veterinaria y Zootecnia. Montería - Córdoba, Colombia; e-mail: morisbustamante@correo. unicordoba.edu.co; (D) http://orcid.org/0000-0003-2874-2025

Cómo citar: Suárez-Paternina, E.; Maza-Angulo, L.; Aguayo-Ulloa, L.; Vergara-Garay, O.; Barragán-Hernández, W.; BustamanteYánez; M. 2020. Efecto de la suplementación con semilla de algodón y maíz en el desempeño productivo y calidad de la carne de ovinos. Rev. U.D.C.A Act. \& Div. Cient. 23(2):e1604. http://doi.org/10.31910/rudca.v23.n2.2020.1604

Artículo de acceso abierto publicado por Revista U.D.C.A Actualidad \& Divulgación Científica, bajo una licencia Creative Commons CC BY-NC 4.0

Publicación oficial de la Universidad de Ciencias Aplicadas y Ambientales U.D.C.A, Institución de Educación Superior Acreditada de Alta Calidad por el Ministerio de Educación Nacional.

Recibido: Junio 27, 2018 Aceptado: Junio 27, 2020 Editado por: Ingeborg Zenner de Polanía

\section{RESUMEN}

La alimentación de los ovinos para producción de carne en la región Caribe colombiana, se fundamenta en el uso de gramíneas, en virtud de sus bajos costos; sin embargo, los rendimientos y la calidad nutricional, se ven afectados por la estacionalidad de las lluvias, por lo que se presentan bajas ganancias de peso. El objetivo de este trabajo fue evaluar el efecto de la suplementación con semilla de algodón y de maíz sobre el desempeño productivo y calidad de la carne de ovinos criollos de pelo. El estudio, se realizó en la Facultad de Medicina Veterinaria y Zootecnia de la Universidad de Córdoba, Ciénaga de Oro-Colombia. Se utilizaron 24 ovinos, distribuidos aleatoriamente en cuatro tratamientos consistentes en: T0: pastoreo; T1: pastoreo $+25 \% \mathrm{SA}+75 \% \mathrm{M}$; $\mathrm{T} 2$ : pastoreo $+50 \mathrm{SA}+50 \%$ M y T3: pastoreo $+75 \% \mathrm{SA}+25 \% \mathrm{M}$. Los animales, se pesaron al inicio de la fase experimental y cada 15 días. Se estudiaron las siguientes variables: peso de la canal caliente y fría, rendimiento canal fría, longitud de la canal, perímetro de pierna, perímetro torácico, 
grasa de cobertura, índice de compacidad, humedad, $\mathrm{pH}$, capacidad de retención de agua y color. La ganancia de peso difirió $(\mathrm{p} \leq 0,05)$ entre tratamiento, presentando valores medios de 0,$053 ; 0,126 ; 0,128$; 0,130kg, para T0, T1, T2 y T3, respectivamente. De igual forma, se detectaron diferencias $(p \leq 0,05)$ para los pesos y rendimientos en canal. Los animales suplementados con $75 \%$ de semilla de algodón y $25 \%$ de maíz generaron las mayores ganancias de peso, viéndose reflejado en un mayor peso al sacrificio y rendimientos.

Palabras clave: Ovinos; Alimentación; Calidad de carne; Ganancia de peso; Rendimiento.

\section{ABSTRACT}

The feeding of sheep for meat production in the Colombian Caribbean Region is based on the use of grasses by virtue of their low costs. However, the yields and nutritional quality are affected by the seasonality of the rains, so there are low weight gains. The objective of this work was to evaluate the effect of the supplementation with cotton seed and corn grain on the productive performance and quality of the meat of sheep creole of Colombian hair. The study was carried out in the the Faculty of Veterinary Medicine and Zootechnics of the University of Córdoba, Ciénaga de Oro-Colombia. Twenty four male creole lambs with initial weight of $16 \pm 2 \mathrm{~kg}$ were used, which were randomly distributed in four experimental treatments consisting of: T0: Grazing, T1: Grazing $+25 \%$ cottonseed (CS) $+75 \%$ corn (C), T2: Grazing $+50 \mathrm{CS}+50 \% \mathrm{C}$ and T3: Grazing $+75 \% \mathrm{CS}+25 \% \mathrm{C}$. Animals were weighed at the start of the experimental phase and every 15 days. The following variables were studied: weight of the hot and cold carcass, performance of the cold carcass, carcass length, leg perimeter, thoracic perimeter, covering fat, compactness index, humidity, $\mathrm{pH}$, water retention capacity and color. The weight gain differed $(\mathrm{p} \leq 0.05)$ between treatment, presenting mean values of $0.053 ; 0.126 ; 0.128 ; 0.130 \mathrm{~kg}$ for T0, T1, T2 and T3, respectively. Similarly, differences $(\mathrm{p} \leq 0.05)$ were detected for carcass weights and yields. The supplementation with cottonseed and corn improved the productive performance of the animals, being reflected in greater gains of weights and yields.

Keywords: Sheep; Feeding; Meat quality; Performance; Weight gain.

\section{INTRODUCCIÓN}

La producción ovina en Colombia, se ha caracterizado por un escaso uso de insumos y un bajo nivel tecnológico en todas las áreas productivas. Generalmente, está asociada a sistemas de producción, tradicionales y artesanales, donde las familias campesinas poseen ovinos o caprinos, como parte de su actividad pecuaria, para convertir desechos de cosecha o forraje de muy mala calidad, en carne, en leche y en lana. Estos sistemas atienden, principalmente, el autoconsumo interno de las granjas y el comercio local, por lo que son poco importantes en su aporte al producto interno bruto del país, pero tienen gran impacto en la economía y alimentación de las familias campesinas (Pastrana, 2002).
Por otro lado, de acuerdo con el Instituto Colombiano Agropecuario (ICA), el inventario ovino nacional, se estimó en 1.629 .120 animales, distribuidos a lo largo del territorio nacional; sin embargo, hay regiones con una mayor actividad, en lo que ovinocultura se refiere, destacándose la región Caribe colombiana, que concentra el 72,8\% del inventario ovino nacional. De igual forma, esta región posee cerca de 6.735.373ha en pastos, la principal fuente de alimento de los ovinos, en virtud de su bajo costo de producción; no obstante, los sistemas ganaderos enfrentan serias limitantes que influyen en la competitividad y la sostenibilidad del sector, debido a las fuertes variaciones en el clima, a través del año, que se manifiestan con períodos de lluvias intensas y períodos de prolongada sequía, con duración aproximada de cuatro a cinco meses, ocasionando disminución en la producción ganadera, principalmente, por la disminución en los rendimientos y la calidad nutricional de las gramíneas, lo que trae pérdidas de peso en los animales (Mejía et al. 2013). En este orden de ideas, los parámetros productivos reportados para ovinos de pelo colombiano no son alentadores, ya que se registran bajos pesos al nacimiento $2,06 \pm 0,4 \mathrm{~kg}$, bajo peso al destete $15,01 \pm 3,5 \mathrm{~kg}$ y bajas ganancias diarias de pesos postdestete $70 \pm$ 0,03g (Roncallo et al. 1999; Maza et al. 2015), lo cual, conlleva a que se presenten edades tardías al sacrificio mayores a los 300 días, bajo peso al sacrificio $(<30 \mathrm{~kg}$ ) y rendimientos en canales inferiores al 50\% (Maza et al. 2015). Por lo anterior, es necesario establecer estrategias en la alimentación ovina; por ello, los ingredientes alimenticios o subproductos agroindustriales, tanto energéticos como proteicos disponibles en la región, pueden contribuir a mejorar las ganancias de pesos y los rendimientos en canales. En este sentido, los granos y las semillas de oleaginosas, como la del algodón y del maíz, representan una excelente fuente de nutrientes para la alimentación de los ovinos.

La semilla de algodón es un subproducto agroindustrial de alto valor nutricional para la alimentación de rumiantes; se caracteriza por contener altas concentraciones de lípidos, proteína y fibra, a un bajo costo; sin embargo, su calidad nutricional se ve afectada por el contenido de gossipol, por lo que su inclusión para dietas en los rumiantes, se debe regular (Akande et al. 2010). Asimismo, otro factor que limita el uso de la semilla de algodón es el alto contenido de extracto etéreo que, dependiendo del nivel utilizado en la dieta, puede afectar la digestibilidad de la fibra. Al respecto, algunos trabajos han evidenciado que la utilización de la semilla de algodón en la suplementación de ovinos ha mejorado el consumo de materia seca, el desempeño productivo y algunas características de la canal (Cunha et al. 2008).

Debido a que en Colombia el sistema de producción de carne de ovino bajo semi-confinamiento no presenta documentación con soporte científico sobre la dinámica de crecimiento, rendimiento y calidad de las canales, se hace necesario realizar este tipo de estudios. Por lo tanto, el objetivo de esta investigación fue evaluar el efecto de la suplementación con semilla de algodón y de maíz grano, sobre el desempeño productivo y calidad de la carne de ovinos criollos de pelo. 


\section{MATERIALES Y MÉTODOS}

Localización: El estudio, se realizó en la granja experimental de la Facultad de Medicina Veterinaria y Zootecnia de la Universidad de Córdoba, sede Berástegui, municipio de Ciénaga de Oro, departamento de Córdoba, Colombia, ubicada entre los 852'41" N y 75³7’27” O, zona catalogada climáticamente como Bosque seco tropical (Bs-T) (Holdridge, 1978), a una altura de $22 \mathrm{~m}$ s.n.m., temperatura promedio de $28^{\circ} \mathrm{C}$, humedad relativa de $85 \%$ y precipitación promedio anual de $1.340 \mathrm{~mm}$; la época de lluvias comprende abril a finales de noviembre, donde se registra, aproximadamente, el 85\% de la precipitación y, la época seca, desde diciembre hasta mediados de abril.

Área experimental y manejo animal: El área utilizada en pastoreo fue $10.000 \mathrm{~m}^{2}$, establecida en un $70 \%$ en Estrella africana (Cynodon nlemfuensis) y en un 30\% de Angleton (Dichantium aristatum), la cual, se dividió en 13 potreros de $713 \mathrm{~m}^{2}$, con el fin de establecer un sistema rotacional de 2 días de ocupación y 24, de descanso. Los ovinos pastorearon en horas de la mañana (08:00h) y retornaban al aprisco en la tarde (16:00h), donde eran separados por tratamiento, para el suministro del suplemento en los comederos y del agua, a voluntad, en bebederos con capacidad de 20 litros. El suplemento mineral, se suministró en los potreros y mezclado con el suplemento en los comederos diariamente.

Animales experimentales y descripción de los tratamientos: Se utilizaron 24 ovinos machos criollos de pelo sin castrar, con un peso inicial de $16 \pm 2 \mathrm{~kg}$ al destete y una edad de 4 meses, adquiridos en fincas cercanas a la Universidad de Córdoba. Además de la edad, se priorizó que los ovinos tuvieran tamaño y condición corporal similar. Antes de iniciar el período de acostumbramiento, los animales fueron sometidos a cuarentena por 20 días y a exámenes coprológicos, para determinar la carga parasitaria.

Las dietas experimentales fueron formuladas de acuerdo con la NRC (2007), atendiendo los requerimientos de proteína y energía metabolizable, para una ganancia diaria de peso de $160 \mathrm{~g}$, para T1, T2 y T3, respectivamente. Los alimentos utilizados para constituir los suplementos fueron semilla de algodón y maíz en grano. La inclusión de la semilla de algodón y maíz en base seca variaron, para así conformar los tratamientos a evaluar. T0: pastoreo, T1: pastoreo $+25 \%$ semilla de algodón $(\mathrm{SA})+75 \%$ maíz $(\mathrm{M})$, T2: pastoreo + $50 \% \mathrm{SA}+50 \% \mathrm{M}$ y T3: pastoreo $+75 \% \mathrm{SA}+25 \% \mathrm{M}$.

Composición química y degradabilidad in situ de la materia seca: La calidad nutritiva del forraje y suplementos, se determinó de muestras compuestas, una vez, durante el ciclo de ceba (Tabla 1 y 2); las muestras de forraje, se colectaron a través del método de simulación de pastoreo (hand plucking) y se colectaron $500 \mathrm{~g}$ por muestra, secadas en estufa de ventilación forzada a $60^{\circ} \mathrm{C}$, por $48 \mathrm{~h}$. Posteriormente, fueron molidas con un molino tipo Wiley, mediante la utilización de una malla de un milímetro y almacenadas hasta su procesamiento. El procesamiento de las muestras, se realizó en el Laboratorio de Nutrición Animal de Agrosavia CI Turipaná, en donde se determinó proteína cruda (método Kjeldahl), fibra en detergente neutro (FDN), fibra en detergente ácido (FDA) y lignina, según AOAC (2002) y la degradabilidad in situ de la materia seca (DISMS), según la técnica de la bolsa de nylon, descrita por Orskov et al. (1980).

Tabla 1. Calidad nutricional de los forrajes utilizados en la alimentación de ovinos criollos.

\begin{tabular}{|c|c|c|c|c|c|c|c|}
\hline Forrajes & MS (\%) & PC (\%) & FDN (\%) & FDA (\%) & EE (\%) & DIGMS (\%) & EM (Mcal/kgMS) \\
\hline C. nlemfuensis & 27,90 & 13,10 & 56,40 & 36,36 & 2,38 & 58,89 & 2,00 \\
\hline Semilla algodón & 89,70 & 26,60 & 63,53 & 44,89 & 18,6 & 55,76 & 2,50 \\
\hline Maíz & 97,90 & 11,20 & 19,55 & 7,71 & 2,74 & 91,9 & 3,20 \\
\hline
\end{tabular}

MS: materia seca, PC: proteína cruda, FDN: fibra detergente neutro, FDA: fibra detergente ácido, EE: extracto etéreo, DIGMS: digestibilidad de la materia seca, EM: energía metabolizable.

Tabla 2. Composición nutricional de las dietas de los tratamientos.

\begin{tabular}{|c|c|c|c|c|}
\hline \multirow{2}{*}{ Componente } & \multicolumn{4}{|c|}{ Composición nutricional de las dietas } \\
\cline { 2 - 5 } & T0 & T1 & T2 & T3 \\
\hline PC (\%) & 13,10 & 14,80 & 15,60 & 16,00 \\
\hline EM Mcal/kg & 2,10 & 2,30 & 2,30 & 2,30 \\
\hline FDN (\%) & 56,40 & 48,60 & 51,90 & 55,20 \\
\hline EE (\%) & 2,40 & 3,62 & 4,90 & 6,10 \\
\hline
\end{tabular}

PC: proteína cruda, EM: energía metabolizable, FDN: fibra detergente neutro, EE: extracto etéreo, T0: pastoreo, T1: pastoreo $+25 \% \mathrm{SA}+75 \%$ maíz M, T2: pastoreo $+50 \% \mathrm{SA}+50 \%$ M y T3: pastoreo $+75 \% \mathrm{SA}+25 \% \mathrm{M}$. 
Para determinar el consumo de materia seca (CMS), se hizo necesario estimar el volumen de heces y la digestibilidad del alimento, para lo cual, se utilizó óxido de cromo, como marcador externo y la fibra detergente ácida indigerible, como marcador interno (Ferreira $e t a l$. 2009). Para la determinación de la concentración del óxido de cromo en las heces, se aplicó la metodología de digestión por miocroondas (3051), propuesta por EPA (2007). La producción fecal estimada en $g \mathrm{MS} / \mathrm{d}^{-1}$ fue obtenida mediante el cociente de las dosis del marcador, dividido por la concentración del marcador en las heces. Con base en la FDAi, se estimó la digestibilidad de la MS, utilizando la ecuación 1, propuesta por Correa et al. (2011).

DMS $=100-100 \times(\%$ de marcador en la MS del alimento $) \times($ tasa de recuperación del marcador en las heces) / (\% del marcador en la MS de las heces)

(Ecuación 1)

El CMS, se determinó en función de la producción fecal y la digestibilidad estimada con el marcador interno; se utilizó la ecuación 2 propuestas por Ramírez-Pérez et al. (2000).

$$
\text { Consumo voluntario }(\mathbf{g} / \mathbf{d})=\frac{\text { (Producción fecal, g MS/ d })}{\left[1-\left(\frac{D i g M S}{100}\right)\right]}
$$

(Ecuación 2)

Los animales, se pesaron al inicio y quincenalmente, hasta el final del ensayo, con báscula electrónica portátil y se determinó la ganancia diaria de peso (GDP). Para la evaluación de la condición corporal, se utilizó una escala de 1 a 5 , teniendo en cuenta la metodología propuesta por Manazza (2012), al inicio y posteriormente cada 15 días, hasta final del ensayo.

Sacrificio y evaluación de la calidad de las canales: Al final del ensayo, los animales se transportaron hasta el frigorífico para ser beneficiados. Previo a esto, los ovinos fueron sometidos a ayuno por un período de 12 horas, con acceso a agua. Después que los animales fueran desollados, eviscerados y lavados, se determinó el peso de canal caliente (PCC) y, luego de permanecer 24 horas, a $4^{\circ} \mathrm{C}$, el peso de canal fría (PCF). Se calculó el rendimiento de canal en caliente y frío, como la relación entre el PCC, PCF y el peso vivo (PV), en ayuno. En la canal fría, se determinó el largo de la canal. El índice de compacidad de la canal, se fijó como el cociente entre el PCF $(\mathrm{kg})$ y la longitud de la canal $(\mathrm{cm})$. El grado de engrasamiento, se estableció a través de la profundidad de los tejidos sobre la $12^{\mathrm{a}}$ costilla, a $11 \mathrm{~cm}$ de la línea media, punto GR (Kirton \& Johnson, 1979). La merma de las canales, se determinó como la diferencia entre el PCC y PCF. Se definió el contenido de humedad. El pH, se midió trascurrido 24 horas post-sacrificio, según la metodología propuesta por SNDA (1999). La capacidad de retención de agua (CRA) muscular, se determinó por el método de compresión entre dos placas de metacrilato, descrito por Cañeque \& Sañudo (2005). En las canales, se fijó el color del músculo, en forma objetiva, a las $36 \mathrm{~h}$ post-sacrificio, utilizando un colorímetro Minolta (C-10), en el espacio CIE Lab, valorando la luminosidad $\left(L^{*}\right)$, el índice de rojo $\left(a^{*}\right)$ y el índice de amarillo $\left(b^{*}\right)$.
Registro de la información y diseño experimental: Para el registro y los análisis de la información, se construyó una base de datos en Microsoft Excel. El diseño estadístico consistió en un diseño completo aleatorizado (DCA), con cuatro tratamientos y seis repeticiones (se consideró cada animal como una repetición). El modelo matemático que describió el diseño fue:

$$
\mathbf{Y}_{\mathrm{ij}}=\mathrm{u}+\mathbf{T}_{\mathrm{j}}+\mathrm{e}_{\mathrm{ij}}
$$

Donde. $Y_{i j}$ es el efecto del tratamiento; $\mu$ es la media general; $T_{j}$ es el efecto del j- ésimo tratamiento; $E$ es el error aleatorio de la ij- ésima repetición que recibió el j-ésimo tratamiento.

Análisis estadístico: Se realizó análisis de varianza (ANOVA), previo cumplimiento de los supuestos de normalidad y de homogeneidad de los datos, para lo cual, se utilizó el tests de Shapiro Wilk y Levene. Para el análisis de los datos, se empleó el procedimiento de modelo lineal generalizado (GLM), del paquete de análisis estadístico SAS V9.2 (SAS Inc. North Carolina, USA). Las medias de tratamiento se compararon, utilizando la prueba de Tukey, con un nivel de significancia de $\mathrm{p} \leq 0,05$.

\section{RESULTADOS Y DISCUSIÓN}

En la tabla 3, se observa que hubo un mayor consumo de materia seca total $(p \leq 0,05)$ en los animales suplementados con respecto a los animales del tratamiento control. Asimismo, se percibió que el consumo de forraje tendió $(\mathrm{p}=0,07)$ a incrementarse en los animales suplementados, sugiriendo que hubo un efecto estimulativo del CMS y de nutrientes. Estos resultados, se pueden atribuir a una mayor digestibilidad y calidad nutricional de las dietas de los tratamientos suplementados (Tabla 1 y 2), respectivamente. La ingestión promedio de MS encontrada en los animales suplementados fue de 1,06kg/día, acorde con los reportados por la NRC (2007), para ovinos de $30 \mathrm{~kg}$ de peso vivo. De la misma forma, Cabrera Núñez et al. (2007) reportaron consumos similares $\left(1,14 \mathrm{~kg}\right.$ animal.d- $\left.{ }^{1}\right)$ en ovinos Dorper x Kathadin, suplementados con pasta de soya y maíz. En cuanto al consumo estimado de PB de los animales de los tratamientos T1, T2 y T3 fueron similares con un valor medio de $0,169 \mathrm{~kg} /$ día, atendiendo la recomendación de la NRC (2007), de $0,167-0,191 \mathrm{~kg} / \mathrm{d}^{-1}$, respectivamente, para corderos de $20-30 \mathrm{~kg}$ de peso vivo. Esta respuesta, probablemente, ocurre en virtud del mayor aporte de la PB que tuvieron las dietas y al aumento en el CMS de los animales de estos tratamientos, lo cual, mejoró el desarrollo de la microflora ruminal y el proceso de fermentación. Similares resultados fueron reportados por Cunha et al. (2008) y Piona et al. (2012), quienes obtuvieron un consumo de PB medio de 0,185 y $0,169 \mathrm{~kg} / \mathrm{d}^{-1}$, respectivamente, en ovinos Santa Inés, suplementados con diferentes porcentajes de inclusión de semilla de algodón.

Con relación al consumo de EM estimado fue menor en los animales del tratamiento control, debido a que provino, principalmente, de las gramíneas. Al respecto, Piona et al. (2012) manifestaron que la energía pasa hacer el principal factor limitante en condiciones tropicales, ya que proviene del FDN de las gramíneas; en este sentido, concentraciones altas de FDN genera que el aporte de 
Tabla 3. Valores medios para los consumos estimados de materia seca y de nutrientes ovinos criollos de pelo colombiano.

\begin{tabular}{|c|c|c|c|c|c|}
\hline Variable & T0 & T1 & $\mathrm{T} 2$ & T3 & $\mathbf{P}$ \\
\hline DIGMS & $47,68 \mathrm{c}$ & $56,23 \mathrm{~b}$ & $58,62 \mathrm{ab}$ & $59,04 \mathrm{a}$ & 0,001 \\
\hline CMST kg/d- ${ }^{1}$ & $0,514 \mathrm{~b}$ & $0,788 \mathrm{ab}$ & $1,02 \mathrm{ab}$ & $1,40 \mathrm{a}$ & 0,0366 \\
\hline CMSF kg/d- ${ }^{1}$ & 0.514 & 0.463 & 0.695 & 1.07 & 0.0750 \\
\hline CMSS kg/d- ${ }^{1}$ & - & 0.325 & 0.325 & 0.325 & - \\
\hline CPB kg/d- ${ }^{1}$ & 0,121 & 0,160 & 0,169 & 0,178 & - \\
\hline CEM Mcal/d- ${ }^{1}$ & 1,96 & 2,53 & 2,44 & 2,36 & - \\
\hline
\end{tabular}

Letras diferentes en las filas difieren estadísticamente, según la prueba de Tukey $(\mathrm{p}<0,05)$. CMST: consumo de materia seca total; CMSF: consumo de materia seca de forraje; CMSS: consumo de materia seca de suplemento; CPB: consumo de proteína bruta; CEM: consumo de energía metabolizable; T0: pastoreo; T1: pastoreo + 25\% SA + 75\% maíz M; T2: pastoreo + 50\% SA + 50\% M y T3: pastoreo + 75\% SA $+25 \%$ M, P: probabilidad.

energía ocurra de forma lenta, lo que limita la tasa de crecimiento de los microorganismos en el rumen. El consumo de EM de los animales que recibieron semilla de algodón y maíz estuvo dentro de los valores propuestos por la NRC (2007), para animales en crecimiento, con ganancias de pesos moderadas; sin embargo, fueron inferiores a los reportados por Cunha et al. (2008), quienes obtuvieron consumos medios de EM de 2,56; 2,69 y 2,84Mcal/día, al incluir en un 20, 30 y 40\% semilla de algodón, respectivamente.

El desempeño productivo de los animales en los diferentes tratamientos puede ser observado en la tabla 4. En este contexto, las ganancias diarias de peso fueron significativamente superiores $(p \leq 0,05)$ en los animales suplementados, que fueron 58,4\% superior a las ganancias obtenidas por los animales del tratamiento control. Estos resultados pueden estar relacionados por un mayor CMS y de nutrientes por parte de los animales de los tratamientos T1, T2 y T3. Las GDP obtenidas en este estudio difieren a las reportadas por Madruga et al. (2008) y Piona et al. (2012), quienes obtuvieron GDP promedio de 0,186 y 0,189kg/día, en corderos Santa Inés, confinados y alimentados con diferentes niveles de semilla de algodón.
En lo que respecta al PCC y PCF, el análisis detectó diferencias significativas $(\mathrm{p} \leq 0,05)$, siendo las canales del tratamiento control las más ligeras (Tabla 5). De igual forma, se encontraron diferencias $(\mathrm{p} \leq 0,05)$ en el rendimiento de las canales, presentando los animales del T2 los mejores rendimientos en canal fría, con un 45,17\%, seguido del T3 y T2. En promedio, los tratamientos que recibieron suplementación con semilla de algodón y maíz fueron superiores al tratamiento control, en un 29, 31,9 y 8,0\%, para PCC, PCF y RCF, respectivamente. Los valores alcanzados en este estudio demuestran que el aporte de nutrientes a partir de la semilla de algodón y de maíz es utilizado con mayor eficiencia para las ganancias de peso, viéndose reflejado en un mayor peso al sacrificio y rendimientos. Hallazgos similares fueron registrados por Pires et al. (2006), quienes obtuvieron PCC y PCF, de $13 \mathrm{~kg}$ y $12,5 \mathrm{~kg}$ respectivamente, en ovinos Santa Inés puros, sacrificados con pesos similares a los obtenidos en esta investigación. Yamamoto et al. (2005), evaluando diferentes fuentes de aceites vegetales en ovinos Santa Inés y Dorset por Santa Inés, reportaron valores PCC y PCF, de 14,5 y $14,1 \mathrm{~kg}$, para Santa Inés y 14,4 y 14,1 kg, para el cruce entre Dorset por Santa Inés, respectivamente, resultados similares a los encontrados en este caso.

Tabla 4. Desempeño productivo de ovinos criollos de pelo suplementados.

\begin{tabular}{|c|c|c|c|c|c|}
\hline Variable & T0 & T1 & T2 & T3 & P \\
\hline Días experimental & 127 & 127 & 127 & 127 & - \\
\hline Peso inicial & $16,50 \mathrm{a}$ & $15,90 \mathrm{a}$ & $15,50 \mathrm{a}$ & $16,80 \mathrm{a}$ & 0,9713 \\
\hline Peso final & $23,30 \mathrm{~b}$ & $32,03 \mathrm{a}$ & $31,78 \mathrm{a}$ & $32,65 \mathrm{a}$ & 0,0063 \\
\hline GDP $(g)$ & $53,60 \mathrm{~b}$ & $126,90 \mathrm{a}$ & $128,10 \mathrm{a}$ & $130,50 \mathrm{a}$ & 0,0004 \\
\hline Condición corporal & $2,0 \mathrm{~b}$ & $3,0 \mathrm{a}$ & $3,0 \mathrm{a}$ & $3,0 \mathrm{a}$ & 0,001 \\
\hline
\end{tabular}

Letras diferentes en las filas difieren estadísticamente, según la prueba de Tukey ( $\mathrm{p} \leq 0,05)$. GDP: ganancia diaria de peso; T0: pastoreo; T1: pastoreo + 25\% SA + 75\% maíz M; T2: pastoreo + 50\% SA + 50\% M y T3: pastoreo + 75\% SA + 25\% M, P: probabilidad. 
Tabla 5. Rendimiento de la canal y calidad de la carne de ovinos criollos de pelo.

\begin{tabular}{|c|c|c|c|c|c|}
\hline Variables & T0 & T1 & T2 & T3 & $\mathbf{P}$ \\
\hline PCC (kg) & $11,00 \mathrm{~b}$ & $15,26 \mathrm{a}$ & $16,03 \mathrm{a}$ & $15,40 \mathrm{a}$ & 0,0098 \\
\hline PCF (kg) & $9,51 \mathrm{~b}$ & $13,54 \mathrm{a}$ & $14,48 \mathrm{a}$ & $13,90 \mathrm{a}$ & 0,0052 \\
\hline RCF (\%) & $39,51 \mathrm{~b}$ & $41,33 \mathrm{ab}$ & $45,17 \mathrm{a}$ & $42,14 \mathrm{ab}$ & 0,029 \\
\hline PR (\%) & $13,5 \mathrm{~b}$ & $11,2 \mathrm{ab}$ & $9,7 \mathrm{a}$ & $9,6 \mathrm{a}$ & 0,0163 \\
\hline $\mathrm{LC}(\mathrm{cm})$ & $53,00 \mathrm{~b}$ & $57,80 \mathrm{a}$ & $58,60 \mathrm{a}$ & $59,00 \mathrm{a}$ & 0,0254 \\
\hline GC (mm) & $0,33 \mathrm{a}$ & $0,29 \mathrm{a}$ & $0,35 \mathrm{a}$ & $0,37 \mathrm{a}$ & 0,6283 \\
\hline IC $\left(\mathrm{kg} / \mathrm{cm}^{3}\right)$ & $0,20 \mathrm{~b}$ & $0,26 \mathrm{a}$ & $0,27 \mathrm{a}$ & $0,26 \mathrm{a}$ & 0,014 \\
\hline Humedad (\%) & $74,88 \mathrm{a}$ & $70,26 \mathrm{a}$ & $69,80 \mathrm{a}$ & $70,26 \mathrm{a}$ & 0,1988 \\
\hline $\mathrm{pH}$ & $5,55 \mathrm{a}$ & $5,62 \mathrm{a}$ & $5,56 \mathrm{a}$ & $5,51 \mathrm{a}$ & 0,1035 \\
\hline CRA \% & $43,43 \mathrm{a}$ & $53,43 \mathrm{a}$ & $46,14 \mathrm{a}$ & $47,03 \mathrm{a}$ & 0,1961 \\
\hline $\mathbf{L}^{*}$ & $41,77 \mathrm{a}$ & $40,38 \mathrm{a}$ & $39,2 \mathrm{a}$ & $42,34 \mathrm{a}$ & 0,2162 \\
\hline$a^{*}$ & $13,07 \mathrm{a}$ & $13,16 \mathrm{a}$ & $14,09 \mathrm{a}$ & $13,14 \mathrm{a}$ & 0,5986 \\
\hline $\mathrm{b}^{*}$ & $15,18 \mathrm{a}$ & $14,98 \mathrm{a}$ & $15,16 \mathrm{a}$ & $15,42 \mathrm{a}$ & 0,1712 \\
\hline
\end{tabular}

Letras diferentes en las filas difieren estadísticamente, según la prueba de Tukey ( $<<0,05)$. PCC: peso canal caliente; PCF: peso canal fría; RCF: rendimiento canal fría; PR: pérdidas por refrigeración; LC: longitud de la canal; GC: grasa de cobertura; IC: índice de compacidad; CRA: capacidad de retención de agua; L*: luminosidad; a*: coordenadas verde-rojo; b*: coordenadas azul-amarillo; T0: pastoreo; T1: pastoreo $+25 \%$ SA $+75 \%$ maíz M; T2: pastoreo + 50\% SA + 50\% M y T3: pastoreo + 75\% SA + 25\% M; P: probabilidad.

Por otra parte, se encontraron diferencias significativas en las pérdidas de peso de las canales, luego de $24 \mathrm{~h}$ de refrigeración ( $\mathrm{p} \leq 0,05)$, las cuales, fueron de 13,$5 ; 11,2 ; 9,7$ y $9,6 \%$, para T0, T1, T2 y T3, respectivamente. Las pérdidas por oreo expresan la diferencia de peso entre PCC y PCF, lo que se relaciona con el contenido de grasa de cobertura de las canales (Sañudo, 1991). En este sentido, los animales del tratamiento control presentaron un menor score corporal al momento del sacrificio, debido al menor aporte de nutrientes recibido; esto pudo haber generado que las canales tuvieran una menor cobertura de grasa e influyera en las pérdidas por refrigeración. De acuerdo con lo anterior, Queiroz et al. (2015) manifestaron que las pérdidas por refrigeración están influenciadas por el espesor de grasa subcutánea, siendo las canales con menor contenido de grasa subcutánea las que presentan las mayores pérdidas. Cunha et al. (2008) reportaron pérdidas por oreo del 2,10\%, valores considerados normales e inferiores a los valores medios $(11,4 \%)$, encontrados en el presente estudio. Según Martins et al. (2000), en el ganado ovino, por lo general, las tasas de pérdida por oreo son, alrededor, del 2,5\%, presentándose oscilaciones entre el 1 y el 7\%, de acuerdo con la uniformidad de la cobertura de grasa, el sexo, el peso, la temperatura y la humedad relativa en el cuarto frío; no obstante, para la grasa de cobertura no se hallaron diferencias significativas $(p>0,05)$ entre los tratamientos, presentando valores medios entre 0,29 y $0,37 \mathrm{~mm}$, siendo inferiores a los emitidos por Fernandes Junior et al. (2013), quienes encontraron valores de 2,66; 2,83; 3,5; y 2,5mm, en ovinos Santa Inés. De igual forma, los resultados hallados en este estudio difieren a los valores registrados por Cunha et al. (2008), quienes evaluaron diferentes porcentajes de inclusión de semilla de algodón en la dieta de ovinos Santa Inés, encontrando valores de 1,17; 1,17 y 1,08mm. Diferencias entre estudios pueden estar relacionadas con el biotipo animal, como también con el sistema de alimentación utilizado.

El índice de compacidad fue mayor $(\mathrm{p}>0,05)$ en las canales provenientes de los animales que recibieron suplementación T1, T2 y T3, los que, además, resultaron ser los más pesados al momento del sacrificio, resultados que se atribuyen a lo anteriormente expresado, con relación al mayor aporte de nutrientes de las dietas de estos tratamientos. El índice de compacidad de la canal para los tratamientos suplementados fue, en promedio, de $0,26 \mathrm{~kg} . \mathrm{cm}^{3}$, lo que coincidió con los reportados por Queiroz et al. (2015), quienes evaluaron canales de ovinos Santa Inés sacrificados con diferentes espesores de grasa subcutánea, registrando valores medios de 0,22; 0,26 y $0,28 \mathrm{~kg} \cdot \mathrm{cm}^{3}$, para los animales sacrificados con 2,3 y $4 \mathrm{~mm}$ de grasa subcutánea. Por otro lado, el estudio de Queiroz et al. (2015) demostró que los animales sacrificados con más grasa subcutánea tienen más tejido muscular y menores pérdidas por refrigeración.

Con relación al contenido de humedad, pH24 y CRA, no se encontró efecto $(\mathrm{p}>0,05)$ de los tratamientos sobre estas variables, presentando valores medios de 71,3; 5,5 y 47,4\%, respectivamente. De igual forma, para el color no se detectaron diferencias $(p>0,05)$ entre los tratamientos, registrando valores medios de 40,9; 13,3 y 15,1, para los índices $\mathrm{L}^{*}, \mathrm{a}^{*} \mathrm{y} \mathrm{b}^{*}$, respectivamente.

Los valores del $\mathrm{pH}$ de las canales estuvieron relativamente constantes, variando entre 5,5 a 5,6. Estos resultados están de conformidad con lo registrado por Silva Sobrinho et al. (2005), quienes obtuvieron valores entre 5,5 y 5,8 cuando sustituyeron harina de soya por semilla de algodón integral en un 40\%, para ovinos Santa Inés. 
Similares hallazgos fueron reportados por Hernández-Cruz et al. (2009), quienes obtuvieron en ovinos Pelibuey un $\mathrm{pH}$, al post mórtem, de 5,80 y 5,53, respectivamente. Por otra parte, Vieira et al. (2010), evaluando las propiedades físicas y sensoriales de la carne de ovinos Santa Inés, terminados con diferentes niveles de semilla de algodón integral, observaron valores de $\mathrm{pH}$ de 5,9; 5,8 y 5,8 para 20, 30 y 40\% de inclusión de semilla de algodón, respectivamente, valores superiores a los encontrados en esta investigación. Los valores de $\mathrm{pH}$ registrados en este ensayo, indican que los animales no fueron sometidos a un nivel de estrés, lo que se pudiera ver reflejado en la calidad de la carne.

No se encontró efecto de los tratamientos sobre la CRA, presentando promedios de 47,5\%. En este sentido, Leão et al. (2012), al evaluar las características físico-químicas y sensoriales de carne de cordero terminados con ensilaje de sorgo y concentrado, reportaron 58,38\%; Sañudo (1991) manifestó que el sistema de producción y la alimentación no afecta la CRA en ovinos; sin embargo, Ramírez et al. (2007) valorando la calidad de la carne y el análisis sensorial en ovinos de pelo y lana provenientes de engorda intensiva en México, encontraron efecto significativo $(\mathrm{p} \leq<0,05)$, con valores medios de 42,4 y $32,2 \%$, respectivamente, similares a los encontrados.

Entre las características físicas de la carne, los parámetros de luminosidad, índice de rojo y de amarillo, no sufrieron efecto $(\mathrm{p}>0,05)$, por las diferentes proporciones de inclusión de la semilla de algodón y de maíz. De acuerdo con lo anterior, se puede inferir que la naturaleza de los alimentos utilizados no ejerció influencia sobre el color de la carne ovina, posiblemente, a que estos sufrieron una intensa trasformación en el rumen. En este contexto, Olleta \& Sañudo (2009) señalan que el color de la carne se puede ver afectado por la raza, la edad, el peso sacrificio, el tipo de músculo, el ejercicio y el proceso de congelación. En Brasil, Vieira et al. (2010), evaluando diferentes porcentajes de inclusión de semilla de algodón en la dieta de ovinos, reportaron valores de $\mathrm{L}^{*}, \mathrm{a}^{*} \mathrm{y} \mathrm{b}^{*}$ inferiores a los encontrados en el presente estudio y anotaron que el color de la carne sufrió un efecto con la inclusión de semilla de algodón, presentando efecto lineal creciente $\left(\mathrm{Y}=17,2+0,05^{*} \mathrm{CAM}, \mathrm{r}^{2}=\right.$ $0,16)$. De este modo, estos autores sugieren que la semilla de algodón molida aumentó la intensidad de L* y a*, en la carne de corderos.

La alimentación con semilla de algodón y de maíz promovió el consumo de materia seca y de nutrientes. Asimismo, mejoró el peso al sacrificio, las ganancias de peso y el rendimiento de las canales.

Conflicto de intereses: El manuscrito fue preparado y revisado con la participación de todos los autores quienes declaramos que no existe conflicto de intereses que ponga en riesgo la validez de los resultados presentados.

\section{REFERENCIAS}

1. AKANDE, K.E.; DOMA, U.D.; AGU, H.O.; ADAMU, H.M. 2010. Major antinutrients found in plant protein sources: their effect on nutrition. Pak. J. Nutr. 9(8):827-832. https://doi.org/10.3923/pjn.2010.827.832
2. AOAC INTERNATIONAL. 2002. Official methods of analysis of the AOAC International. 17th edition current through 1st revision. Gaithersburg: Association of Analytical Communities.

3. CABRERA NÚÑEZ, A.; ROJAS MENCIO, P.; DANIEL RENTERIA, L.; SERRANO SOLIS, A.; LÓPEZ ORTEGA, M. 2007. Influencia de la suplementación sobre la ganancia de peso y calidad de la canal en borregos Dorper/Katahdin. Revista UDO Agrícola. 7(1):245-251.

4. CAÑEQUE, V.; SAÑUDO, C. 2005. Estandarización de las metodologías para evaluar la calidad del producto (animal vivo, canal, carne y grasa en los rumiantes). Ed. Instituto Nacional de Investigación y Tecnología Agraria y Alimentaria. Madrid, España. 448p.

5. CORREA, H.J.; PABÓN, M.L.; SANCHÉZ, M.Y.; CARULLA, J.E. 2011. Efecto del nivel de suplementación sobre el uso del nitrógeno, el volumen y la calidad de la leche en vacas Holstein de primero y segundo tercio de lactancia en el trópico alto de Antioquia. Livestock Research for Rural Development. 23(4):77.

6. CUNHA, M. DAS GRACAS GOMES.; CARVALHO, F.F. RAMOS DE; GONZAGA NETO, S.; CEZAR, M.F. 2008. Características quantitativas de carcaça de ovinos Santa Inês confinados alimentados com rações contendo diferentes níveis de caroço de algodão integral. Rev. Brasileira de Zootecnia. 37(6):1112-1120. https://doi.org/10.1590/S1516-35982008000600023

7. EPA. 2007. Method 3051A (SW-846): Microwave Assisted Acid Digestion of Sediments, Sludges, and Oils," Revision 1. Washington, DC. 30p.

8. FERNANDES JUNIOR, F;; RIBEIRO, E.L.A.; MIZUBUTI, I.Y.; SILVA, L.D.F.; BARBOSA, M.A.A.F.; PRADO, O.P.P.; PEREIRA, E.S.; PIMENTEL, P.G.; CONSTANTINO, C. 2013. Características de carcaça e qualidade da carne de cordeiros Santa Inês alimentados com torta de girassol em substituição ao farelo de algodão. Semina: Ciências Agrárias. 34(6):3999-4014. http://dx.doi.org/10.5433/1679-0359.2013v34n6Supl2p3999

9. FERREIRA, M. DE ANDRADE; VALADARES FILHO, S. DE CAMPOS; MACONDOS, M.I.; PAIXÃO, M. LOPES; PAULINO, M. FONSECA; VALADARES, R. FERREIRA DINIZ. 2009. Avaliação de indicadores em estudos com ruminantes: digestibilidade. Rev. Brasileira Zootecnia. 38(8):1568-1573.

https://dx.doi.org/10.1590/S1516-35982009000800022

10. HERNÁNDEZ-CRUZ，L.; RAMÍREZ-BRIBIESCAI, J.E.; GUERRERO-LEGARRETAII, M.I.; HERNÁNDEZ-MENDOI, O.; CROSBY-GALVAN, 
M.M.; HERNÁNDEZ-CALVA, L.M. 2009. Effects of crossbreding on carcass and meat quality of Mexican lambs. Arq. Bras. Med. Vet. Zoo. 61:475-483.

http://dx.doi.org/10.1590/S0102-09352009000200027

11. HOLDRIDGE R. 1978. Capítulo 2. El diagrama de las zonas de vida. Ecología basada en zonas de vida. San José: Instituto Interamericano de Cooperación para la Agricultura (IICA). p.13-28.

12. KIRTON, A.; JOHNSON, D. 1979. Interrelationships between GR and other lamb carcass fatness measurements. Proc. New Zealand Soc. Anim. Prod. 39:194-201.

13. LEÃO, A.G.; SILVA SOBRINHO, A.G. DA; BEZERRA MORENO, G.M.; ALVES DE SOUZA, H.B.; GIAMPIETRO, A.; ROSSI, R.C.; LEAL PEREZ, H. 2012. Características físico-químicas e sensoriais da carne de cordeiros terminados com dietas contendo cana-deaçúcar ou silagem de milho e dois níveis de concentrado. Rev. Brasileira Zootecnia. 41(5):1253-1262. https://doi.org/10.1590/S1516-35982012000500024

14. MADRUGA, M.S.; VIEIRA, T.R. DE L.; CUNHA, M. DAS G.G.; PEREIRA FILHO, J.M.; QUEIROGA, R. DE C.R.E.; SOUSA, W.H. DE. 2008. Efeito de dietas com níveis crescentes de caroço de algodão integral sobre a composição química e o perfil de ácidos graxos da carne de cordeiros Santa Inês. Rev. Brasileira Zootecnia. 37(8):14961502. https://doi.org/10.1590/S1516-35982008000800023

15. MANAZZA, J. 2012. Condición corporal en ovinos. Disponible desde Internet en:

https://www.agrositio.com.ar/noticia/62154-condicioncorporal-en-ovinos (con acceso 12/08/2018).

16. MARTINS, R.R.C.; OLIVEIRA, N.M. DE; OSÓRIO, J.C. DA S.; OSÓRIO, M.T.M. 2000. Peso vivo ao abate como indicador do peso e das características quantitativas e qualitativas das carcaças em ovinos jovens da raça Ideal. Bagé: Embrapa Pecuária Sul. 29p.

17. MAZA ANGULO, L.; BUSTAMANTE YANEZ, M.; SIMANCA SOTELO, J.C.; RUIZ ORTIZ, M.; MONTAÑO FLÓREZ, G.; VERGARA GARAY, O. 2015. Efecto de la suplementación sobre la ganancia de peso y rendimiento en canal de corderos Sudan. Rev. U.D.C.A Act. \& Div. Cient. 18(1):283-286.

https://doi.org/10.31910/rudca.v18.n1.2015.448

18. MEJÍA KERGUELÉN, S.; CUADRADO CAPELLA, H.; RIVERO ESPITIA, T. 2013. Manejo agronómico de algunos cultivos forrajeros y técnicas de conservación en la región Caribe Colombiana. Corporación Colombiana de Investigación Agropecuaria. 2a ed. Bogotá, Colombia. 77p.
19. NATIONAL RESEARCH COUNCIL, NRC. 2007. Nutrient requirements of small ruminants: sheep, goats, cervids, and New World camelids. Washington: National Academic Press. 362p.

20. OLLETA, J.; SAÑUDO, C. 2009. La carne ovina. En: Sañudo, C.; Cepero, R. (eds). Ovinotecnia: producción y economía en la especie ovina. Zaragoza: Prensa Universitaria de Zaragoza. p.327-336.

21. ORSKOV, E.; HOWELL, F.; MOULD, F. 1980. The use of nylon bag technique for the evaluation of feedstuff. Trop. Anim. Prod. 15(3):195-213.

22. PASTRANA, R. 2002. Ovinocultura colombiana: su origen y evolución. En: García Alzate, H.; Parra López, L.G. (eds). Medicina veterinaria y zootecnia en Colombia, trayectoria durante el siglo XX y perspectivas para el siglo XXI. 1 ed. Bogotá: Edivez. 875p.

23. PIONA, M.N.M.; CABRAL, L. DA S.; ZERVOUDAKIS, J.T.; ABREU, J.G. DE; GALATI, R.L.; CAETANO, G.G. DE P.; SILVA, A.R. DA. 2012. Niveis de Caroço de algodão na dieta de cordeiros confinados. Rev. Bras. Saúde Prod. Anim. 13(1):110-122.

https://dx.doi.org/10.1590/S1519-99402012000100010

24. PIRES, C.C.; GALVANI, D.B.; CARVALHO, S.; CARDOSO, A.R.; GASPERIN, B.G. 2006. Características da carcaça de cordeiros alimentados com dietas contendo diferentes níveis de fibra em detergente neutro. Rev. Brasileira Zootecnia. 35(5):2058-2065. https://dx.doi.org/10.1590/S1516-35982006000700025

25. QUEIROZ, L. DE O.; SANTOS, G.R. DE A.; MACÊDO, F. DE A.F.; MORA, N.H.A.P.; TORRES, M.G.; SANTANA, T.E.Z.; MACÊDO, F.G. DE. 2015. Características quantitativas da carcaça de cordeiros Santa Inês, abatidos com diferentes espessuras de gordura subcutánea. Rev. Bras. Saúde Prod. Anim. 16(3):712-722. http://dx.doi.org/10.1590/S1519-99402015000300021

26. RAMÍREZ, B.; HERNÁNDEZ, C.; GUERRERO, L.; HERNÁNDEZ, C. 2007. Calidad de la carne y análisis sensorial en ovinos de pelo y lana provenientes de engorda intensiva en México. Sitio Argentino de Producción Animal. Disponible desde Internet en:

http://www.produccion-animal.com.ar/produccion_ ovina/produccion_ovina_carne/113-ramiez.pdf (con acceso el 05/07/2019).

27. RAMÍREZ-PÉREZ, A.H.; BUNTINX, S.E.; TAPIARODRÍGUEZ, C.; ROSILES, R. 2000. Effect of breed and age on the voluntary intake and the micromineral status 
of non-pregnant sheep. 1. Estimation of voluntary intake. Small Ruminant Research. 37:223-229.

https://doi.org/10.1016/S0921-4488(99)00141-8

28. RONCALLO, B.; TOLOZA, A.; BARROS, J.; SILVA, J.; ARAUJO, A.; MEJIA, M.; AVILA, E.; ROBLEDO, M. 1999. Sistema de producción ovino-caprinos en los departamentos de la Guajira, Cesar y Magdalena. CORPOICA. 52p.

29. SAÑUDO, C. 1991. La calidad organoléptica de la carne con especial referencia a la especie ovina. Factores que la determinan, métodos de medidas y causas de variación. In: curso internacional sobre producción de ganado ovino, Zaragoza. 117p.

30. SECRETARIA NACIONALDE DEFESA AGROPECUÁRIA (SNDA). 1999. Métodos analíticos físico-químicos para controle de produtos cárneos e seus ingredientes: sal e salmoura. Instrução normativa no 20. Brasília. 4p.
31. SILVA SOBRINHO, A.G. DA.; KADIM, I.T; YAMAMOTO, S.M. 2005. Características de qualidade da carne de ovinos de diferentes genótipos e idades ao abate. Rev. Brasileira de Zootecnia. 34(3):1070-1078.

https://doi.org/10.1590/S1516-35982005000300040

32. VIEIRA, T.R DE L.; CUNHA, M. DAS G.G.; GARRUT'TI, D. DOS S.; DUARTE, T.F.; FÉLEX, S.S DOS S.; PEREIRA, FILHO, J.M.; MADRUGA, M.S. 2010. Propriedades físicas e sensoriais da carne de cordeiros Santa Inês terminados em dietas com diferentes níveis de caroço de algodão integral (Gossypium hirsutum). Ciência e Tecnologia de Alimentos. 30(2):372-377.

https://doi.org/10.1590/S0101-20612010000200013

33. YAMAMOTO, S.M.; MACEDO, F. DE A.F. DE M.; ZUNDT, M.; MEXIA, A.A.; SAKAGUTI, E.S.; ROCHA, G.B.L.; REGAÇONI, K.C.T.; MACEDO, R.M.G. DE G. 2005. Fontes de óleo vegetal na dieta de cordeiros em confinamento. Rev. Brasileira Zootecnia. 34(2):703-710. https://doi.org/10.1590/S1516-35982005000200040 